\title{
Research on 3D printed fixture components
}

\author{
Dragoș-Florin Chitariu*, and Adriana Munteanu \\ Machine-tools and Tools Department, Technical University "Gheorghe Asachi” of Iasi, Prof Dr. Doc. \\ D. Mangeron Street 67, Iasi, Romania
}

\begin{abstract}
Fixtures are used for orientation, positioning and tightening of the workpiece during machining, control and assembly. The main fixture requirements are: orientation, positioning and tightening precision in accordance with the machining requirements. The materials used for fixture components, especially, supports and clamping mechanism are, usually, alloy steel with HRC hardness up to 55-60 HRC. These components are machined to high level of precision thus assuring the overall precision of the fixture. In order to achieve high stiffness and a good dampening capacity the fixture become, usually, very heavy. In the case of manually operated fixtures light weight is an advantage; also there are operations such as inspection, assembly where the operating forces are low. In this case lightweight materials can be used for fixture construction. In this paper the FDM (Fused Deposition Modeling) 3D printing technology is used. Support buttons and v-block fixture components were selected and 3D printed. The effect of printing orientation of active surfaces of support was analysed. The dimensional accuracy and surface roughness on the active surface were measured. Experimental results indicate that surface roughness is dependent on the orientation of the printed workpiece.
\end{abstract}

\section{Introduction}

Fixtures are used for orientation, positioning and tightening of the workpiece during machining, control and assembly. The main fixture requirements are: orientation, positioning and tightening precision in accordance with the machining requirements, rigidity in accordance to the size of the workpiece and machining forces, reliability, low maintenance, standardized components, high productivity, low cost [1-5].

In order to ensure the fulfillment of the functions the materials used in the construction of fixtures are usually alloy steel with HRC hardness up to 55-60 HRC for fixture components used for both orientation and positioning of the workpiece or self-centering mechanism, clamping mechanism, and other components found in the structure (e.g. fixture base plate, braces, frame) [1-3].

Fixture components are machined to high level of precision, thus assuring the overall precision of the fixture, high stiffness good dampening capacity, maintaining workpiece stability under the influence of the static and dynamic mechanical loads generated by the machining processes. Such that fixture become, usually, very heavy. In the case of manually

\footnotetext{
* Corresponding author: chitariudragos@gmail.com
} 
operated fixtures light weight is an advantage; also there are operations such as inspection, assembly where the operating forces are low. Also, there are cases where "soft" jaws/ supports are needed in order to reduce workpiece deformations, especially for thin walled workpieces [6].

As the Additive Manufacturing technology evolve manufacturer of such specialized equipment present as an application field, alongside prototypes, replacement parts, $3 \mathrm{~d}$ printing of fixture and fixture components. It should be noted that most of the examples provided are for inspection and assembly fixtures, less for machining fixtures [7-9].

Additive manufacturing (AM) is a process of joining materials to make parts from $3 \mathrm{D}$ model data, usually layer upon layer, as opposed to subtractive manufacturing and formative manufacturing methodologies. Additive manufacturing is the formalized term for what used to be called rapid prototyping and the production process called 3D Printing [10].

The objective of this paper is to identify components of devices that are suitable for 3D printing, to manufacture a series of components and analyze their accuracy and compare it to the regular, standardized fixture components.

\section{Methodology}

To accomplish a research on the 3D Printed Fixture Components, the following methodology was suggested:

1. Selection of the studied fixture components;

2. Identification of accuracy characteristics for selected components;

3. Manufacturing of fixture components;

4. Measuring of accuracy characteristics and data processing.

5. Analysis and interpretation of the results.

\subsection{Selection of the studied fixture components}

By considering the fixture component classification proposed by professor Gherghel, the general structure of technological fixtures consist of at least 35 group of components, of these, there are common components to all fixtures and specific to various types of fixtures [2].

The common components are: 1. elements, mechanisms, subassemblies for orientation and position of the workpiece (supports, locators); 2. clamping components used for tightening the workpiece during machining, having the role of converting and distributing the acting force, 3 . self-centering components - that simultaneous centering and clamping the workpiece; 4. actuation elements, drive components that are prime mover for the clamping mechanism, 5. internal connexion components, frame; 6 . components for fixture locating in the technological system; 7. components for fixture clamping in the technological system; 8. assembly components [2].

In this paper fix supports were selected in the form of support button with plain surface, hexagonal head, exterior threaded and fixed v-block [11]. The support button selected is manufactured by Erwin Halder KG and the v-block is from the Tolerance and Control Laboratory inventory. The selected fixed supports being presented in figure 1 .

\subsection{Identification of accuracy characteristics for selected components}

The recommendation from fixture design literature regarding dimensioning, tolerances, materials and treatments consider as the main requirements imposed on the supports are: ensuring the required orientation-positioning precision; mechanical resistance; not to damage 
the orientation-positioning surfaces of the parts; easy insertion and removal of all parts of the batch; high wear resistance; ease of fabrication; low cost.

By taking into account these requirements for the selection of materials, the following criteria will also be taken into consideration: support type: fixed or extensible; support size; required accuracy; force magnitude; clamping frequency; physical and mechanical properties; lifetime; - cost.
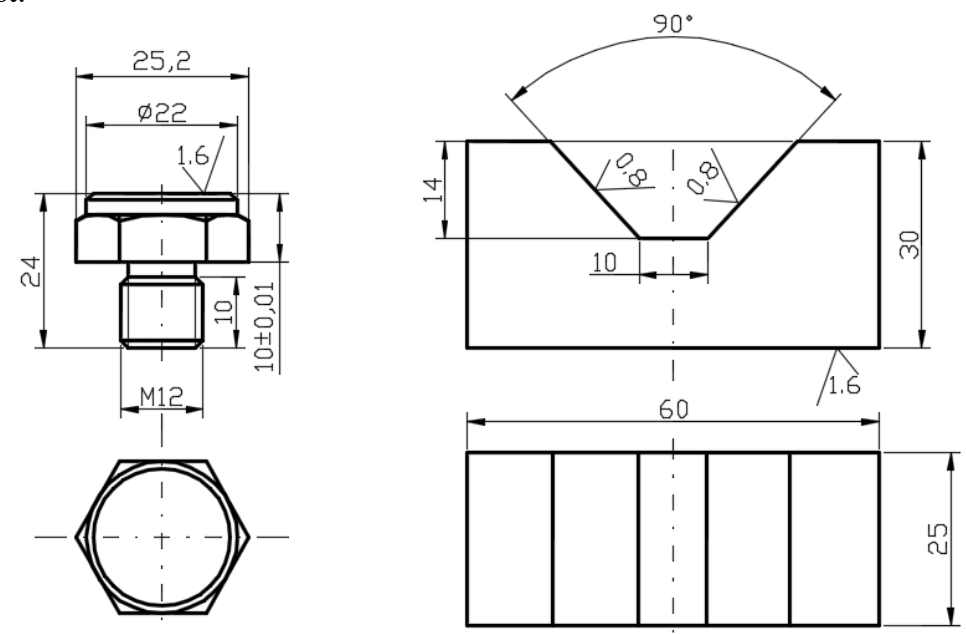

Fig. 1. Selected fixed supports.

For support manufacturing the following steel are recommended: Quenched and tempered steels - 1C45, 2C45, 3C45 and Case hardening steels 25CrMo4, 34CrMo4, 30CrNiMo8.

Dimensioning is done according to constructively-functional considerations and is done by choice or adoption or by calculations based on specific indications.

Tolerance classes 4-14 may be used to choose the tolerance dimensions of supports. For free dimensions, tolerances of class $\mathrm{mK}$ are used [1-3].

\subsection{Manufacturing of fixture components}

The selected supports were modeled in SolidWorks CAD software, after finishing the model, conversion was made in the STL format, with the fine option.

The preprocessing of the STL model was realized in the 3D printer Insight command software for selecting the appropriate printing parameters.

The printer used is Stratasys Fortus $250 \mathrm{mc}$, that uses Fused Deposition Modeling (FDM) technology to build parts from the bottom up with precisely deposited layers of modeling and support material, with the following features: ABS plus P-430 - production-grade thermoplastic and soluble support material, build envelope - 254 × 254 × $305 \mathrm{~mm}$, three layer thicknesses $0.178,0.254$ and $0.330 \mathrm{~mm}$.

Tensile strength yield for ABS P430 thermoplastic is $31 \mathrm{MPa}$ (xz axis), $26 \mathrm{MPa}$ (zx axis).

Extruded plastic has its strongest strength in the tensile mode along the $x-y$ plane. Since the layers are held together by "hot flow" across the strands (one strand is cooling while the other is laid upon it), the lowest strength is in the Z-direction for both tensile and shear modes.

Printing parameters were selected according to the recommendation from speciality literature and considering the machine maximum possibilities [12-15]:

1. parts slicing (on $\mathrm{z}$ axis): $0.254 \mathrm{~mm}$.

2. visible surface raster: $0.3556 \mathrm{~mm}$;

3. internal raster: $0.3556 \mathrm{~mm}$;

4. raster angle: 0 ; 
5. part raster width: 0.3556 .

By considering the presented parameters and the recommendations for orientation of the printed parts regarding, printing speed, part strength, surface finish and material consumption, the following orientations were selected for the fixed supports.

In Table 1 the printing orientation of the v-blocks is presented. Considering the presented orientation 1 and 2 , the effect of layers that form the contours of the exterior surfaces on the surface roughness can be evaluated.

Table 1. Printing orientation of v-blocks.

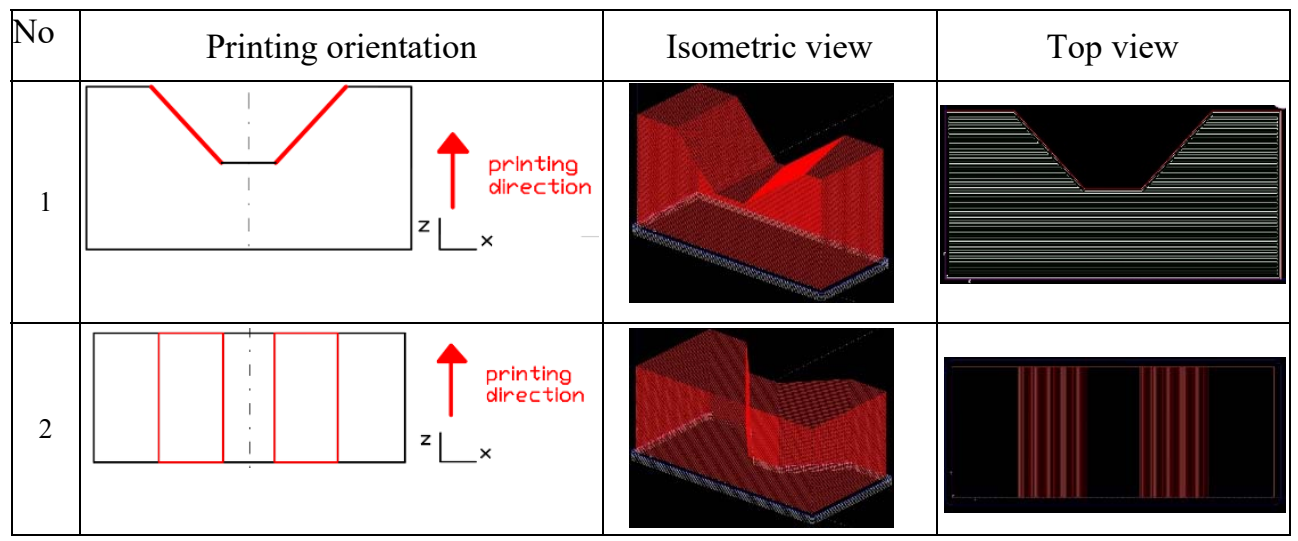

In Table 2 the printing orientation of support buttons with plain surface and hexagonal head are presented. The effect of printing the support with vertical (1) or horizontal axis (2) can be evaluated and also, the contact of the active surface with the support material (3).

Table 2. Printing orientation of support button with plain surface and hexagonal head.



Roughness results of the active surfaces on two directions are presented in Table 3 for $\mathrm{v}$ blocks and in Table 4 for support buttons. Roughness was measured with Mitutoyo Portable Surface Roughness Measurement Surftest SJ-210 In Tables 3 and 4 the printing direction is 
marked and the active surfaces of the support are highlighted. The isometric view and top view present the extrusion path in darker colors and the support material in lighter color

\section{Results}

The printed parts are presented in figure 2 and 3.

By following the dimensional measurements, all parts were found to be correct, by considering the imposed precision.



Fig. 2. Steel and thermoplastic v-blocks.

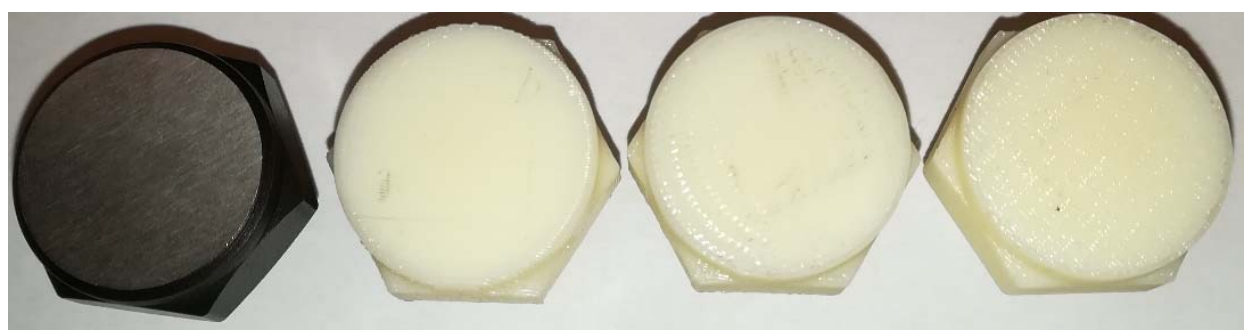

Fig. 3. Steel and thermoplastic support buttons with plain surface and hexagonal head.

Table 3. Roughness results on the active surfaces on two directions for v-blocks.

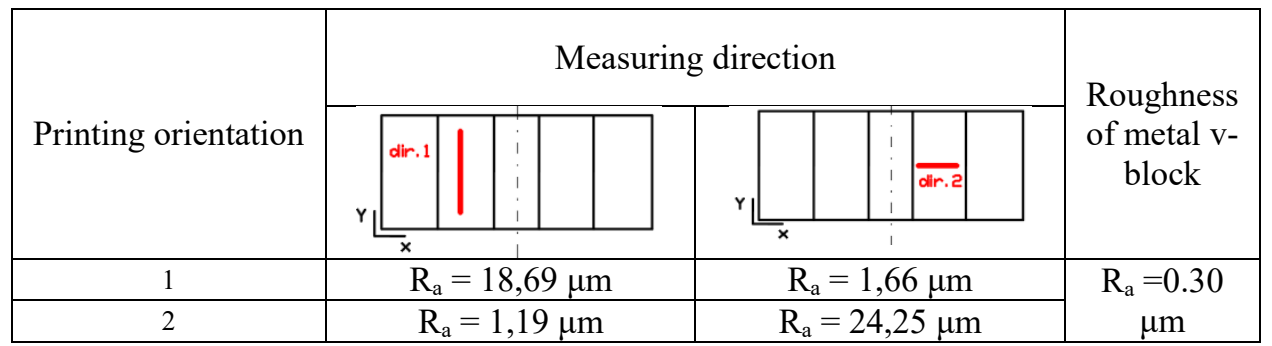

Table 4. Roughness results on the active surfaces on two directions for support buttons.

\begin{tabular}{|c|c|c|c|}
\hline \multirow{2}{*}{ Printing orientation } & \multicolumn{2}{|c|}{ Measuring direction } & \multirow{2}{*}{$\begin{array}{c}\text { Roughness } \\
\text { of metal } \\
\text { support } \\
\text { button }\end{array}$} \\
\cline { 2 - 3 } & & & \\
\hline 1 & $\mathrm{R}_{\mathrm{a}}=1,54 \mu \mathrm{m}$ & $\mathrm{R}_{\mathrm{a}}=8,74 \mu \mathrm{m}$ & \multirow{2}{*}{$\mathrm{R}_{\mathrm{a}}=0,8$} \\
$\mathrm{R}$ & $\mathrm{R}_{\mathrm{a}}=18,74 \mu \mathrm{m}$ & \\
\hline 2 & $\mathrm{R}_{\mathrm{a}}=8,98 \mu \mathrm{m}$ & $\mathrm{R}_{\mathrm{a}}=25,53 \mu \mathrm{m}$ & \\
\hline 3 & & & \\
\hline
\end{tabular}

Roughness was measured on two directions: 1. on the direction of the extrusion path (direction 1) and 2. on the transverse direction (direction 2). From the presented tables the best surface quality was obtained along the extrusion path for both support type. 
Best surface quality was obtained for the button printed on orientation 1, but it was inferior to the one of metal. Surface roughness is influenced by the contact with the support material. The lowest surface quality from all printed support was obtained for the button printed in contact with the support material. Surface roughness is influenced by the visible raster and slice height setting of the printer; in the case of button 2 (Table 4) the measured roughness on transversal direction is over $200 \%$ higher than in the case of button 1 . Roughness is not dependent on surface angle; there are no major differences between a flat surface and angled active surface of the v-block.

\section{Conclusions}

Fixture components made through additive technologies can be used in assembly or in machining processes, where precision conditions are not high and process forces are low. From the presented experimental results, the surface roughness is dependent on the orientation of the printed workpiece; best results are obtained when the active surfaces are included in $\mathrm{x}-\mathrm{y}$ plane and along the extrusion path. Surface roughness is influenced; also, by the visible surface raster setting of the $3 \mathrm{D}$ printer, in all printed parts the minimum value was used. The orientation of the surface raster has large impact on the roughness, as follows, on the extrusion direction the measured roughness is 6-12 times lower than on transversal direction. The angle of the active surface does not have a large impact on the surface roughness. Future research directions will extend the analysis on other fixture components such as jaw for self-centering fixtures, and fixture components with higher precision conditions and performing rigidity, wear, testing of fixture components.

\section{References}

1. N. Gherghel, Construction and Exploitation of Fixtures 1-2, (in Romanian) (Inst. Politehnic Iasi Publishing House, 1981)

2. N. Gherghel, N. Seghedin, Conception and Design of Supports of Technological fixtures (in Romanian) (Tehnopress Publishing House, Iasi, 2006)

3. N. Seghedin, Fixtures (PIM Publishing House, Iasi, 2008)

4. D. F. Chitariu, App. Mech. and Mat., 809-810, (2015)

5. D. F. Chitariu, MATEC Web Conf., 112, 07024, (2017)

6. G. Sridhar, P. Babu, APEM, 10, (2015)

7. 3D printed jigs fixtures powerful solution production floor, Www.stratasysdirect.com/applications accessed 14.10.2017

8. 3D printing tools, jigs and fixtures, www.ultimaker.com/en/explore/how-is-3d-printingused/tools-jigs-fixtures accessed 14.10.2017

9. Secures a Firm Grip in Robotic Automation, www.markforged.com/case-study/themark-two-secures-a-firm-grip-in-robotic-automation/ accessed 14.10.2017

10. Additive manufacturing. General principles. Terminology, ISO/ASTM 52900:2015

11. Halder Standard Parts Catalogue. www.halder.com/de/Produkte/Normalien/

12. A. Munteanu, D. Chitariu, F. Cioata, A. M. M., 809-810, IMANE (2015)

13. T. Grimm, User Guide to Rapid Prototyping, (Society of Manufacturing Engineer, Michigan, 2004)

14. L. N. Marcincinova, Manuf. and Ind. Eng., 11(4), (2012)

15. L. N. Marcincinova, J., Janak, KMM, 581, (2014) 